\title{
How to Construct Mutually Orthogonal Complementary Sets with Non-Power-of-Two Lengths?
}

\author{
Shing-Wei Wu (吳星蔚), Chao-Yu Chen (陳昭羽), Member, IEEE, and Zilong Liu (劉子龍), Member, IEEE
}

\begin{abstract}
Mutually orthogonal complementary sets (MOCSs) have received significant research attention in recent years due to their wide applications in communications and radar. Existing MOCSs which are constructed based on generalized Boolean functions (GBFs) mostly have lengths of power-of-two. How to construct MOCSs with non-power-of-two lengths whilst having large set sizes is a largely open problem. With the aid of GBFs, in this paper, we present new constructions of such MOCSs and show that the maximal achievable set size is $1 / 2$ of the flock size of an MOCS.
\end{abstract}

Index Terms-Golay complementary set (GCS), mutually orthogonal complementary set (MOCS), complete complementary code (CCC), generalized Boolean function.

\section{INTRODUCTION}

The concept of Golay complementary pair (GCP), where the sums of aperiodic autocorrelations of two sequences are zero everywhere except at the in-phase position, was introduced by Marcel J. E. Golay in his design of infrared multislit spectrometry [1]. In 1972, Tseng and Liu generalized the concept to Golay complementary sets (GCSs) and mutually orthogonal complementary sets (MOCSs) [2]. Specifically, each GCS is composed of two or more sequences whose aperiodic autocorrelation sums display "impulse-like" correlation properties as GCP; MOCSs may be viewed as a collection of GCSs in which any two distinct GCSs are mutually orthogonal in terms of their zero cross-correlation sums for all the time-shifts. In 1988, Suehiro and Hatori proposed complete complementary codes (CCC) whose set size (denoted by $M$ ) achieves the upper bound of MOCSs (i.e., $M \leq N$, where $N$ refers to the number of constituent sequences in an MOCS, called the flock size) [3]. Due to the ideal auto- and crosscorrelation properties, MOCSs (and CCCs) have been adopted as a key component for next generation multicarrier code division multiple access (MC-CDMA) systems [4]-[8]. Other

This work of S.-W. Wu and C.-Y. Chen was supported in part by the Ministry of Science and Technology, Taiwan, R.O.C., under Grant MOST 107-2221-E-006-065-MY2. The work of Z. Liu was supported in part by the National Natural Science Foundation of China through the Research Fund for International Young Scientists under Grant 61750110527.

Chao-Yu Chen and Shing-Wei Wu are with the Department of Engineering Science, National Cheng Kung University, Tainan 701, Taiwan, R.O.C. (email: \{super, n98084016\}@mail.ncku.edu.tw).

Zilong Liu is with the School of Computer Science and Electronic Engineering, University of Essex, UK, (e-mail: zilong.liu@essex.ac.uk).

Copyright (c) 2017 IEEE. Personal use of this material is permitted. However, permission to use this material for any other purposes must be obtained from the IEEE by sending a request to pubspermissions@ieee.org. applications include optimal channel estimation in multipleinput and multiple-output (MIMO) frequency-selective channels [9], MIMO radar [10], [11], cell search in orthogonal frequency division multiplexing (OFDM) systems [12], and data hiding [13], et al.

A primary approach to construct MOCSs is based on generalized Boolean functions (GBFs). This was initiated by the landmark work of Davis and Jedwab in [14] which shows that $2^{h}$-ary GCPs lie in certain second-order cosets of the first-order Reed-Muller (RM) codes. Subsequently, Paterson generalized Davis and Jedwab's methods to connect $q$-ary (for even $q$ ) GCPs with GBFs of degree 2 [15]. In the literature, GCPs obtained on GBFs given in [14], [15] are sometimes called the Golay-Davis-Jedwab (GDJ) pairs [16][18]. For GCSs, further constructions of GCSs based on GBFs of high degrees have been proposed in [19]-[22]. In [23], [24], connections of CCCs, MOCSs and cosets of the first-order RM codes have been proposed.

Existing complementary sequences constructed based on GBFs are mostly limited to lengths with powers of two. Recently, some research attempts have been made by Chen for GCSs with more flexible lengths [25]-[27]. GCSs with flexible lengths are more suitable for practical OFDM systems where the number of data subcarriers is various. With the aid of GBFs, however, how to construct MOCSs with non-powerof-two lengths whilst having large set size remains largely open, to the best of our knowledge. Although some MOCS constructions based on a series of sequence operations (e.g., reversals, negations, interleaving, and concatenations) have been proposed in [2], they are unable to give rise to MOCSs with large set sizes. Moreover, these sequence operations may not be friendly for efficient hardware generations. By contrast, the sequences from GBFs have algebraic structures and hence could have efficient encoding algorithms. In [28][30], Das et al. presented methods by using paraunitary (PU) matrices to construct MOCSs and CCCs with various lengths. PU matrices are powerful tools but 1) the available MOCSs heavily depend on the existence of PU matrix kernels and 2) the generations of MOCSs require multiple blocks in serial to carry out complicated PU matrix multiplications. Therefore, the MOCSs based on PU matrices and those based on GBFs might be different.

In this paper, we attack the aforementioned open problem by using GBFs. Such constructions are attractive due to their small storage requirement as well as fast generation capability [31]. For given flock size $N$, we define $r \triangleq N / M$ as the 
performance ratio which is upper bounded by one. In the case of power-of-two lengths, $r=1$ can be achieved by CCC; For non-power-of-two lengths, it is intriguing to know the maximum achievable $r$. By constructing MOCSs with lengths $2^{\theta}+2^{\gamma}$ (where $\theta \neq \gamma$ are positive integers) ${ }^{1}$, it is found that $r=1 / 2$ is achievable.

The remainder of the paper is organized as follow. In Section II, we give the definitions and some notations that will be used throughout this paper. Then we present the several constructions of GCSs and MOCSs with flexible lengths based on GBFs in Section III. Finally, we summarize our paper in Section IV.

\section{PRELIMINARIES AND DEFINITIONS}

\section{A. Correlation Functions}

For two $L$-length sequences $\boldsymbol{c}=\left(c_{0}, c_{1}, \ldots, c_{L-1}\right)$ and $\boldsymbol{d}=$ $\left(d_{0}, d_{1}, \ldots, d_{L-1}\right)$ over $\mathbb{Z}_{q}=\{0,1, \ldots, q-1\}$. The aperiodic cross-correlation function of $\boldsymbol{c}$ and $\boldsymbol{d}$ at time-shift $u$ is defined as

$$
\rho(\boldsymbol{c}, \boldsymbol{d} ; u)= \begin{cases}\sum_{k=0}^{L-1-u} \xi^{c_{k+u}-d_{k}}, & 0 \leq u \leq L-1 \\ \sum_{k=0}^{L-1+u} \xi^{c_{k}-d_{k-u}}, & -L+1 \leq u<0\end{cases}
$$

where $\xi=e^{2 \pi \sqrt{-1} / q}$. When $\boldsymbol{d}=\boldsymbol{c}$, the correlation $\rho(\boldsymbol{c} ; u) \triangleq$ $\rho(\boldsymbol{c}, \boldsymbol{c} ; u)$ refers to the aperiodic autocorrelation function of c.

\section{B. Golay Complementary Set and Mutually Orthogonal Com- plementary Set}

Consider a set of $M$ sequence sets $\mathcal{C}=\left\{C^{p}, p=\right.$ $0,1, \ldots, M-1\}$ where each sequence set $C^{p}$ contains $N$ sequences $C^{p}=\left\{\boldsymbol{c}_{0}^{p}, \boldsymbol{c}_{1}^{p}, \ldots, \boldsymbol{c}_{N-1}^{p}\right\}$ and each sequence has length $L$.

Definition 1: The set $\mathcal{C}$ is called a mutually orthogonal complementary set (MOCS), denoted by $(M, N, L)$-MOCS, if

$$
\begin{gathered}
\rho\left(C^{p_{1}}, C^{p_{2}} ; u\right)=\sum_{n=0}^{N-1} \rho\left(\boldsymbol{c}_{n}^{p_{1}}, \boldsymbol{c}_{n}^{p_{2}} ; u\right) \\
= \begin{cases}N L, & u=0, p_{1}=p_{2} \\
0, & 0<|u|<L, p_{1}=p_{2} \\
0, & |u|<L, p_{1} \neq p_{2}\end{cases}
\end{gathered}
$$

where $M$ is the set size, $N$ the flock size, and $L$ the sequence length. In the above definition, each sequence set $C^{p}$ is called a Golay Complementary Set, denoted by $(N, L)$-GCS. In addition, any two distinctive GCSs in the set $\mathcal{C}$ are said to be mutually orthogonal.

Lemma 2: For a $(M, N, L)$-MOCS, the upper bound on set size satisfies the inequality

$$
M \leq N .
$$

When $M=N$, it is called a Complete Complementary Code (CCC) [32].

${ }^{1}$ See Corollary 5 in Section III for details.

\section{Generalized Boolean Functions (GBFs)}

A GBF $f$ is a function of $m$ variables $x_{1}, x_{2}, \ldots, x_{m}$ which maps any binary vector $\left(x_{1}, x_{2}, \ldots, x_{m}\right)$ in $\mathbb{Z}_{2}^{m}$ to a value in $\mathbb{Z}_{q}$ [33]. We define a product of $r$ variables, $x_{i_{1}} x_{i_{2}} \cdots x_{i_{r}}$, to be a Boolean monomial of degree $r$. For example, $x_{1} x_{2} x_{3}$ is a monomial of degree 3 . We list all the monomials as follows:

$1, x_{1}, x_{2}, \ldots, x_{m}, x_{1} x_{2}, x_{1} x_{3}, \ldots, x_{m-1} x_{m}, \ldots, x_{1} x_{2} \cdots x_{m}$

For $q=2$, any Boolean function $f$ can be uniquely expressed as a linear combination of these $2^{m}$ monomials, where the coefficient of each monomial belongs to $\mathbb{Z}_{2}$ [34]. The resulting expression for $f$ is called the algebraic normal form. Then, we can define a $2^{m}$-length sequence $\boldsymbol{f}=\left(f_{0}, f_{1}, \ldots, f_{2^{m}-1}\right)$ for any $\operatorname{GBF} f$. The $i$ th element of $\boldsymbol{f}$ is given by $f_{i}=$ $f\left(i_{1}, i_{2}, \ldots, i_{m}\right)$ where $\left(i_{1}, i_{2}, \ldots, i_{m}\right)$ is the binary representation of the integer $i=\sum_{j=1}^{m} i_{j} 2^{j-1}$. For example, for $f=x_{1} x_{2}+3$ with $m=3$ and $q=4$, we have the sequence $\boldsymbol{f}=(3,3,3,0,3,3,3,0)$.

For a GBF $f$ with $m$ variables, the corresponding sequence $\boldsymbol{f}$ defined above is of length $2^{m}$. Considering the sequence of length $\neq 2^{m}$, we define the corresponding truncated sequence $\boldsymbol{f}^{(L)}$ of the GBF $f$ by removing the last $2^{m}-L$ elements of the sequence $\boldsymbol{f}$ [25]. That is, $\boldsymbol{f}^{(L)}=\left(f_{0}, f_{1}, \ldots, f_{L-1}\right)$ is a sequence of length $L$ with $f_{i}=f\left(i_{1}, i_{2}, \ldots, i_{m}\right)$ for $i=0,1, \ldots, L-1$ and $\left(i_{1}, i_{2}, \ldots, i_{m}\right)$ is the binary representation of $i$. For example, if taking $m=3$, and GBF $f=x_{1} x_{2}+1$, we have the corresponding truncated sequence $\boldsymbol{f}^{(6)}=(1,1,1,0,1,1)$ and $\boldsymbol{f}^{(7)}=(1,1,1,0,1,1,1)$. For the sake of simplicity, the superscript of $\boldsymbol{f}^{(L)}$ is disregarded when the length $L$ can be determined from the context.

\section{Constructions of MOCSs with Flexible LENGTHS}

In this section, we first provide a construction of GCSs based on GBF. Then we extend the construction to MOCSs of non-power-of-two lengths.

Theorem 3: For any positive integers $m$ and $k$ where $m \geq 2$ and $k \leq m$, we consider a partition of $\{1,2, \ldots, m-1\}$ denoted by $I_{1}, I_{2}, \ldots, I_{k}$. Let $\pi_{\alpha}$ be a bijection from $\mathbb{N}_{m_{\alpha}}=$ $\left\{1,2, \ldots, m_{\alpha}\right\}$ to $I_{\alpha}$ where $m_{\alpha}$ is the order of $I_{\alpha}$ for $\alpha=$ $1,2, \ldots, k$. Let the GBF

$$
f=\frac{q}{2} \sum_{\alpha=1}^{k} \sum_{\beta=1}^{m_{\alpha}-1} x_{\pi_{\alpha}(\beta)} x_{\pi_{\alpha}(\beta+1)}+\sum_{l=1}^{m} g_{l} x_{l}+g_{0},
$$

where $q$ is an even integer and $g_{l} \in \mathbb{Z}_{q}$ for $l=0,1, \ldots, m$. For a positive integer $t$ with $0 \leq t \leq m-1$, if $t \neq 0$, then there exist integers $\alpha^{\prime}$ and $\beta^{\prime}$ such that $t=\sum_{\alpha=1}^{\alpha^{\prime}-1} m_{\alpha}+\beta^{\prime}$ where $1 \leq \beta^{\prime} \leq m_{\alpha^{\prime}}$. Note that if $t \leq m_{1}$, we let $\alpha^{\prime}=1$ and $\beta^{\prime}=t$. When $t \neq 0$, we need an additional condition below.

$$
\begin{gathered}
\left\{\pi_{1}(1), \pi_{1}(2), \ldots, \pi_{1}\left(m_{1}\right), \pi_{2}(1), \ldots, \pi_{2}\left(m_{2}\right),\right. \\
\left.\pi_{3}(1), \ldots, \pi_{\alpha^{\prime}}(1), \ldots, \pi_{\alpha^{\prime}}\left(\beta^{\prime}\right)\right\}=\{1,2, \ldots, t\} .
\end{gathered}
$$

Then, $C=\left\{\boldsymbol{c}_{0}, \boldsymbol{c}_{1}, \ldots, \boldsymbol{c}_{2^{k+1}-1}\right\}$ is a $\left(2^{k+1}, 2^{m-1}+2^{t}\right)$-GCS where

$$
\boldsymbol{c}_{n}=\boldsymbol{f}+\frac{q}{2}\left(\sum_{\alpha=1}^{k} n_{\alpha} \boldsymbol{x}_{\pi_{\alpha}(1)}+n_{k+1} \boldsymbol{x}_{m}\right),
$$


and $\left(n_{1}, n_{2}, \ldots, n_{k+1}\right)$ is the binary representations of $n$ for $n=0,1, \ldots, 2^{k+1}-1$.

Proof: The proof is given in Appendix A.

Compared with [25] and [26], the proposed GCSs can exist more lengths by setting a generalized variable $t$ in Theorem 3 . In addition, the constructed GCSs from Theorem 3 can include the results in [25], [26]. For example, Theorem 3 includes [25, Th. 6] as a special case when $t=0$. In addition, if we let $0 \leq t \leq m_{1}$, Theorem 3 can be reduced to [26]. Then we will illustrate the relationship between Theorem 3 and the results in [25], [26] via an example.

In the following example and from here onwards, we let $\pi\left(a_{1}, a_{2}, \cdots, a_{k}\right)$ represent a mapping such that $\pi(1)=a_{1}$, $\pi(2)=a_{2}, \cdots$, and $\pi(k)=a_{k}$.

Example 1: Taking $q=4, m=6$, and $k=2$, we let $I_{1}=$ $\{1,2\}, I_{2}=\{3,4,5\}, \pi_{1}=(1,2), \pi_{2}=(3,4,5)$, and then the Boolean function is $f=2 x_{1} x_{2}+2 x_{3} x_{4}+2 x_{4} x_{5}$ by letting all $g_{l}$ 's $=0$ in (3). Let us consider the case $t=2$, we have $\alpha^{\prime}=1$ and $\beta^{\prime}=2$. Since $\left\{\pi_{1}(1), \pi_{1}(2)\right\}=\{1,2\}$ satisfying (4), the set $C=\left\{\boldsymbol{f}+2 n_{1} \boldsymbol{x}_{1}+2 n_{2} \boldsymbol{x}_{3}+2 n_{3} \boldsymbol{x}_{6}: n_{i} \in \mathbb{Z}_{2}\right\}$ is an $(8,36)$ GCS from Theorem 3. The set $C$ can also be constructed by [26]. Next, if we take $t=3, t$ can be expressed as $t=m_{1}+1$. Hence, we have $\alpha^{\prime}=2$ and $\beta^{\prime}=1$. The condition (4) still holds since $\left\{\pi_{1}(1), \pi_{1}(2), \pi_{2}(1)\right\}=\{1,2,3\}$. Therefore, we can obtain another $(8,40)$-GCS from Theorem 3 . This $(8,40)$ GCS is listed as follows:

$$
\begin{aligned}
C=\{ & \left.\boldsymbol{c}_{0}, \boldsymbol{c}_{1}, \boldsymbol{c}_{2}, \boldsymbol{c}_{3}, \boldsymbol{c}_{4}, \boldsymbol{c}_{5}, \boldsymbol{c}_{6}, \boldsymbol{c}_{7}\right\} \\
=\{ & (0002000200022220000200022220000200020002), \\
& (0200020002002022020002002022020002000200), \\
& (0002222000020002000222202220222000022220), \\
& (0200202202000200020020222022202202002022), \\
& (0002000200022220000200022220000222202220), \\
& (0200020002002022020002002022020020222022), \\
& (0002222000020002000222202220222022200002), \\
& (0200202202000200020020222022202220220200)\} .
\end{aligned}
$$

In this case, [26] cannot construct this $(8,40)$-GCS. Furthermore, if taking $t=0$, [25, Th. 6], [26, Th. 4] and Theorem 3 all give the same $(8,33)$-GCS.

Next, we construct multiple GCSs based on Theorem 3 with mutual orthogonal cross-correlation property, that is, a MOCS.

Theorem 4: We follow the same notations given in Theorem 3 with $f$ defined in (3). For any positive integer $t$ with $m_{1} \leq$ $t \leq m-1$, we can obtain integers $\alpha^{\prime}$ and $\beta^{\prime}$ such that $t=$ $\sum_{\alpha=1}^{\alpha^{\prime}-1} m_{\alpha}+\beta^{\prime}$ where $\alpha^{\prime} \geq 1$ and $1 \leq \beta^{\prime} \leq m_{\alpha^{\prime}}$. Note that $\alpha^{\prime}=1$ and $\beta^{\prime}=m_{1}$ if $t=m_{1}$. Let $k^{\prime}$ be the largest integer satisfying $\sum_{\alpha=1}^{k^{\prime}} m_{\alpha} \leq t$. If

$$
\begin{gathered}
\left\{\pi_{1}(1), \pi_{1}(2), \ldots, \pi_{1}\left(m_{1}\right), \pi_{2}(1), \ldots, \pi_{2}\left(m_{2}\right),\right. \\
\left.\pi_{3}(1), \ldots, \pi_{\alpha^{\prime}}(1), \ldots, \pi_{\alpha^{\prime}}\left(\beta^{\prime}\right)\right\}=\{1,2, \ldots, t\},
\end{gathered}
$$

then the set $\mathcal{C}=\left\{C^{0}, C^{1}, \cdots, C^{2^{k^{\prime}}-1}\right\}$ where $C^{p}=$ $\left\{c_{0}^{p}, c_{1}^{p}, \ldots, c_{2^{k+1}-1}^{p}\right\}$,

$\boldsymbol{c}_{n}^{p}=\boldsymbol{f}+\frac{q}{2}\left(\sum_{\alpha=1}^{k} n_{\alpha} \boldsymbol{x}_{\pi_{\alpha}(1)}+n_{k+1} \boldsymbol{x}_{m}+\sum_{\alpha=1}^{k^{\prime}} p_{\alpha} \boldsymbol{x}_{\pi_{\alpha}\left(m_{\alpha}\right)}\right)$, for $n=0,1, \ldots, 2^{k+1}-1$ and $p=0,1, \ldots, 2^{k^{\prime}}-1$, $\left(n_{1}, n_{2}, \ldots, n_{k+1}\right)$ and $\left(p_{1}, p_{2}, \ldots, p_{k^{\prime}}\right)$ are the binary representations of $n$ and $p$, respectively, is a $\left(2^{k^{\prime}}, 2^{k+1}, 2^{m-1}+2^{t}\right)$ MOCS.

Proof: The proof is given in Appendix B.

Please note that $k^{\prime}=\alpha^{\prime}$ if $\beta^{\prime}=m_{\alpha^{\prime}}$ and $k^{\prime}=\alpha^{\prime}-1$ if $\beta^{\prime}<m_{\alpha^{\prime}}$

Example 2: Following the same parameters $q, m, k$, permutations $\pi_{1}, \pi_{2}$ and Boolean function $f$ given in Example 1, we obtain an $(8,40)$-GCS $C^{0}=C$ in (5). According to Theorem 4 , we have $k^{\prime}=1$ since $m_{1}=2 \leq t$ and $m_{1}+m_{2}=2+3>t$ when $t$ is taken as 3 . As a result, we can obtain a $(2,8,40)$ MOCS from Theorem 4. In addition to $C^{0}$, another constituent GCS is $C^{\prime}=\left\{\boldsymbol{f}+2 n_{1} \boldsymbol{x}_{1}+2 n_{2} \boldsymbol{x}_{3}+2 n_{3} \boldsymbol{x}_{6}+2 \boldsymbol{x}_{2}: n_{i} \in \mathbb{Z}_{2}\right\}$ and given by

$$
\begin{aligned}
C^{1}=\{ & \left.\boldsymbol{c}_{0}^{1}, \boldsymbol{c}_{1}^{1}, \boldsymbol{c}_{2}^{1}, \boldsymbol{c}_{3}^{1}, \boldsymbol{c}_{4}^{1}, \boldsymbol{c}_{5}^{1}, \boldsymbol{c}_{6}^{1}, \boldsymbol{c}_{7}^{1}\right\} \\
=\{ & (0002000200022220000200022220000200020002), \\
& (0200020002002022020002002022020002000200), \\
& (0002222000020002000222202220222000022220), \\
& (0200202202000200020020222022202202002022), \\
& (0002000200022220000200022220000222202220), \\
& (0200020002002022020002002022020020222022), \\
& (0002222000020002000222202220222022200002), \\
& (0200202202000200020020222022202220220200)\} .
\end{aligned}
$$

Similarly, for $t=4$, we can obtain a $(2,8,48)$-MOCS.

From Theorem 4 , we can obtain a $\left(2^{k^{\prime}}, 2^{k+1}, 2^{m-1}+2^{t}\right)$ MOCS where $m_{1} \leq t \leq m-1$. Additionally taking $t=m-1$, the constructed MOCSs are reduced to CCCs of length $2^{m}$. Theorem 4 provides MOCSs of more lengths. Next, we discuss the set size of the constructed MOCSs with length $2^{m-1}+2^{t}$. Let $M_{o}$ denote the optimal set size of the constructed MOCS, i.e., $M_{o}=2^{k+1}$. For $m_{k-1} \leq t<m-1$, we only have set size $2^{k^{\prime}}=2^{\alpha^{\prime}-1}=2^{k-1}$. This means that the set size is $2^{k-1} / M_{o}=1 / 4$ which is one fourth of the optimal set size. Therefore, we propose a new corollary for expanding the set size of MOCSs.

Corollary 5: We follow the same notations given in Theorem 3. If $t=\sum_{\alpha=1}^{k-1} m_{\alpha}+\beta^{\prime}$ for some positive integer $\beta^{\prime} \leq m_{k}$, we let

$$
\boldsymbol{y}=\boldsymbol{x}_{m} \boldsymbol{x}_{\pi_{k}\left(\beta^{\prime}\right)}+\left(\mathbf{1} \oplus \boldsymbol{x}_{m}\right) \boldsymbol{x}_{\pi_{k}\left(m_{k}\right)} .
$$

Then, $\mathcal{C}=\left\{C^{0}, C^{1}, \cdots, C^{2^{k}-1}\right\}$ is a $\left(2^{k}, 2^{k+1}, 2^{m-1}+2^{t}\right)$ MOCS where $C^{p}=\left\{c_{0}^{p}, c_{1}^{p}, \ldots, c_{2^{k+1}-1}^{p}\right\}$ and

$c_{n}^{p}=\boldsymbol{f}$

$+\frac{q}{2}\left(\sum_{\alpha=1}^{k} n_{\alpha} \boldsymbol{x}_{\pi_{\alpha}(1)}+n_{k+1} \boldsymbol{x}_{m}+\sum_{\alpha=1}^{k-1} p_{\alpha} \boldsymbol{x}_{\pi_{\alpha}\left(m_{\alpha}\right)}+p_{k} \boldsymbol{y}\right)$,

for $n=0,1, \ldots, 2^{k+1}-1$ and $p=0,1, \ldots, 2^{k}-1$. Note that $\left(n_{1}, n_{2}, \ldots, n_{k+1}\right)$ and $\left(p_{1}, p_{2}, \ldots, p_{k}\right)$ are the binary representations of $n$ and $p$, respectively.

Proof: The proof is given in Appendix C.

From Corollary 5, the set size of MOCSs is $2^{k}$. This means that the ratio of the set size over the flock size is $M / N=1 / 2$. 
TABLE I

THE EXISTENCE OF MOCS FOR VARIOUS LENGTHS

\begin{tabular}{|c||c|c|c|c|c|c|c|c|c|c|c|}
\hline Length & 4 & 8 & 12 & 16 & 20 & 24 & 32 & 36 & 40 & 48 & 64 \\
\hline 4 [24] & $\sqrt{ }$ & $\sqrt{ }$ & - & $\sqrt{ }$ & - & - & $\sqrt{ }$ & - & - & - & $\sqrt{ }$ \\
\hline 4 (Th. 4 and Cor. 5) & - & $\sqrt{ }$ & $\sqrt{ }$ & $\sqrt{ }$ & $\sqrt{ }$ & $\sqrt{ }$ & $\sqrt{ }$ & $\sqrt{ }$ & $\sqrt{ }$ & $\sqrt{ }$ & $\sqrt{ }$ \\
\hline \hline 8 [24] & - & $\sqrt{ }$ & - & $\sqrt{ }$ & - & - & $\sqrt{ }$ & - & - & - & $\sqrt{ }$ \\
\hline 8 (Th. 4 and Cor. 5) & - & - & - & $\sqrt{ }$ & - & $\sqrt{ }$ & $\sqrt{ }$ & - & $\sqrt{ }$ & $\sqrt{ }$ & $\sqrt{ }$ \\
\hline \hline 16 [24] & - & - & - & $\sqrt{ }$ & - & - & $\sqrt{ }$ & - & - & - & $\sqrt{ }$ \\
\hline 16 (Th. 4 and Cor. 5) & - & - & - & - & - & - & $\sqrt{ }$ & - & - & $\sqrt{ }$ & $\sqrt{ }$ \\
\hline
\end{tabular}

TABLE II

The EXISTEnCE Of EVEN-LENGTh $(M, N)$-MOCS

\begin{tabular}{|c|c|c|c|c|c|c|c|c|c|c|c|c|c|c|}
\hline Construction & 4 & 6 & 8 & 10 & 12 & 16 & 18 & 20 & 24 & 32 & 34 & 36 & 40 & $M / N$ \\
\hline \multirow{4}{*}{$\begin{array}{l}{[23]} \\
{[24]}\end{array}$} & $(4,4)$ & - & $(4,4)$ & - & - & $(4,4)$ & - & - & - & $(4,4)$ & - & - & - & 1 \\
\hline & - & - & $(8,8)$ & - & - & $(8,8)$ & - & - & - & $(8,8)$ & - & - & - & 1 \\
\hline & - & $\begin{array}{cc}- \\
-\end{array}$ & - & - & - & $(16,16)$ & - & - & - & $(16,16)$ & $\begin{array}{lll}- & \\
- & & l\end{array}$ & - & - & 1 \\
\hline & - & - & - & - & - & - & - & - & - & $(32,32)$ & - & - & - & 1 \\
\hline \multirow{13}{*}{ Theorem 4} & $(2,4)$ & - & $(2,4)$ & - & - & $(2,4)$ & - & - & - & $(2,4)$ & - & - & - & $1 / 2$ \\
\hline & - & $(2,8)$ & - & $(2,8)$ & $(2,8)$ & - & $(2,8)$ & $(2,8)$ & $(2,8)$ & - & $(2,8)$ & $(2,8)$ & $(2,8)$ & $1 / 4$ \\
\hline & - & - & - & $(2,16)$ & - & - & $(2,16)$ & $(2,16)$ & - & - & $(2,16)$ & $(2,16)$ & $(2,16)$ & $1 / 8$ \\
\hline & - & - & - & - & - & - & $(2,32)$ & - & - & - & $(2,32)$ & $(2,32)$ & - & $1 / 16$ \\
\hline & - & $\begin{array}{lll}- & & \end{array}$ & $\begin{array}{lll}- & & \end{array}$ & - & - & - & - & - & - & - & $(2,64)$ & - & $\begin{array}{lll}- & & \end{array}$ & $1 / 32$ \\
\hline & - & - & $(4,8)$ & - & - & $(4,8)$ & - & - & - & $(4,8)$ & - & - & - & $1 / 2$ \\
\hline & $\begin{array}{llll}- & & & \\
\end{array}$ & - & - & - & $(4,16)$ & - & - & $(4,16)$ & $(4,16)$ & - & - & $(4,16)$ & $(4,16)$ & $1 / 4$ \\
\hline & - & - & - & - & - & - & - & $(4,32)$ & - & - & - & $(4,32)$ & $(4,32)$ & $1 / 8$ \\
\hline & - & - & - & - & - & - & - & - & - & - & - & $(4,64)$ & - & $1 / 16$ \\
\hline & - & - & - & - & - & $(8,16)$ & - & - & - & $(8,16)$ & - & - & - & $1 / 2$ \\
\hline & - & - & - & - & - & - & - & - & $(8,32)$ & - & - & - & $(8,32)$ & $1 / 4$ \\
\hline & - & - & - & - & - & - & - & - & - & - & - & - & $(8,64)$ & $1 / 8$ \\
\hline & - & - & - & - & - & - & - & - & - & $(16,32)$ & - & - & - & $1 / 2$ \\
\hline \multirow{4}{*}{ Corollary 5} & $(2,4)$ & $(2,4)$ & $(2,4)$ & $(2,4)$ & $(2,4)$ & $(2,4)$ & $(2,4)$ & $(2,4)$ & $(2,4)$ & $(2,4)$ & $(2,4)$ & $(2,4)$ & $(2,4)$ & $1 / 2$ \\
\hline & - & - & $(4,8)$ & - & $(4,8)$ & $(4,8)$ & - & $(4,8)$ & $(4,8)$ & $(4,8)$ & - & $(4,8)$ & $(4,8)$ & $1 / 2$ \\
\hline & - & - & - & - & - & $(8,16)$ & - & - & $(8,16)$ & $(8,16)$ & - & - & $(8,16)$ & $1 / 2$ \\
\hline & - & - & - & - & - & - & 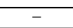 & - & - & $(16,32)$ & - & - & - & $1 / 2$ \\
\hline
\end{tabular}

Table I shows the existences of MOCSs of set size 4,8 and 16 for various lengths. Compared with CCCs from [24], Theorem 4 and Corollary 5 can construct MOCSs of nonpower-of-two lengths. For example, it can be observed that the MOCSs of set size 4 are available for lengths 18, 20, 24 between $2^{4}$ and $2^{5}$.

Example 3: Let us consider the same parameter setting in Example 2. Following the construction in Corollary 5, let $C^{2}=\left\{\boldsymbol{f}+2 n_{1} \boldsymbol{x}_{1}+2 n_{2} \boldsymbol{x}_{3}+2 n_{3} \boldsymbol{x}_{6}+2 \boldsymbol{y}: n_{i} \in \mathbb{Z}_{2}\right\}$ and $C^{3}=\left\{\boldsymbol{f}+2 n_{1} \boldsymbol{x}_{1}+2 n_{2} \boldsymbol{x}_{3}+2 n_{3} \boldsymbol{x}_{6}+2 \boldsymbol{x}_{2}+2 \boldsymbol{y}: n_{i} \in \mathbb{Z}_{2}\right\}$ where $\boldsymbol{y}=\boldsymbol{x}_{6} \boldsymbol{x}_{\pi_{2}(1)}+\left(\mathbf{1} \oplus \boldsymbol{x}_{6}\right) \boldsymbol{x}_{\pi_{2}(3)}=\boldsymbol{x}_{6} \boldsymbol{x}_{3}+\left(\mathbf{1} \oplus \boldsymbol{x}_{6}\right) \boldsymbol{x}_{5}$. Then $\left\{C^{0}, C^{1}, C^{2}, C^{3}\right\}$ forms a $(4,8,48)$-MOCS.

Example 4: Taking $q=2, m=6$ and $k=3$, we let $I_{1}=\{1\}, I_{2}=\{2,4\}, I_{3}=\{3,5\}, \pi_{1}=(1), \pi_{2}=(4,2)$, $\pi_{3}=(3,5)$, and then the Boolean function is $f=x_{4} x_{2}+x_{3} x_{5}$ by letting $g_{l}=0$ for all $l$ in (3). Let us consider the case $t=4$, we have $\alpha^{\prime}=3$ and $\beta^{\prime}=1$ since $t=4=m_{1}+m_{2}+1$. Due to $\left\{\pi_{1}(1), \pi_{2}(1), \pi_{2}(2), \pi_{3}(1)\right\}=\{1,4,2,3\}$, the set $\mathcal{C}=$ $\left\{C^{0}, C^{1}, \cdots, C^{7}\right\}$ is an $(8,16,48)$-MOCS from Corollary 5 where $C^{p}=\left\{\boldsymbol{f}+n_{1} \boldsymbol{x}_{1}+n_{2} \boldsymbol{x}_{4}+n_{3} \boldsymbol{x}_{3}+n_{4} \boldsymbol{x}_{6}+p_{1} \boldsymbol{x}_{1}+\right.$ $\left.p_{2} \boldsymbol{x}_{2}+p_{3}\left(\boldsymbol{x}_{6} \boldsymbol{x}_{3}+\left(\mathbf{1} \oplus \boldsymbol{x}_{6}\right) \boldsymbol{x}_{5}\right): n_{i} \in \mathbb{Z}_{2}\right\}$ and $\left(p_{1}, p_{2}, p_{3}\right)$ is the binary representation of $p$.

In Table II, MOCSs of lengths between 4 and 40 are listed. Theorem 4 and Corollary 5 can construct MOCSs of nonpower-of-two lengths but the set sizes can not be equal to the individual flock sizes. The ratio of the set size over the flock size is $M / N=1 / 2$ for the MOCSs obtained by Corollary 5 .

\section{Conclusion}

In this paper, we have presented new constructions of GCSs and MOCSs based on GBFs with flexible lengths. First, a construction of GCSs with flexible lengths is proposed in Theorem 3, which includes the results in [25], [26] as special cases. In addition, new constructions of MOCSs have been proposed in Theorem 4 and Corollary 5. The resultant MOCSs can be obtained directly from GBFs without using other tedious sequence operations.

The proposed MOCSs have lengths of the form $2^{m-1}+2^{t}$ where $t>0$. Possible future research includes the study of MOCSs with more available lengths, e.g., $t=0$. In Corollary 5 , the ratio of set size over flock size (i.e., $M / N$ ) is $1 / 2$ only. It would be interesting to know that whether CCCs (i.e., $M / N=1$ ) with non-power-of-two lengths can be constructed by GBFs.

\section{APPENDIX A \\ PROOF OF THEOREM 3}

Before starting the proof of Theorem 3, we introduce several lemmas which can be regarded as crucial tools to prove our main theorems. We follow the same notations as given in Theorem 3 and define the common notations as follows. Let two nonnegative integers $i, j<2^{m}$ have binary representations $\left(i_{1}, i_{2}, \ldots, i_{m}\right)$ and $\left(j_{1}, j_{2}, \ldots, j_{m}\right)$, respectively.

Lemma 6: [25, Lemma 2] For two integers $i$ and $j$ with $0 \leq i<j<2^{m}$ and $m \geq 2$, if $i_{s}=j_{s}$ for $s=1,2, \ldots, t$ for a positive integer $t$, then $j \geq i+2^{t}$. 
Lemma 7: [25, Lemma 3] For an integer $i$ with $2^{m-1} \leq$ $i \leq 2^{m-1}+2^{t}-1$ where $1 \leq t \leq m-1$ and $m \geq 2$, if $i^{\prime}$ is an integer with binary representation $\left(i_{1}, i_{2}, \ldots, i_{r-1}, 1-\right.$ $\left.i_{r}, i_{r+1}, \ldots, i_{m}\right)$ and $r \leq t$, then we have $2^{m-1} \leq i^{\prime} \leq$ $2^{m-1}+2^{t}-1$.

Lemma 8: [35, Lemma 4] Following the same definitions and notations given in Theorem 3. If $i_{\pi_{\alpha}(1)} \neq$ $j_{\pi_{\alpha}(1)}$ for some $\alpha \in\{1,2, \ldots, k\}$, then for any sequences $\boldsymbol{c}_{n}=\left(c_{n, 0}, c_{n, 1}, \ldots, c_{n, L-1}\right) \in C$, there exists $\boldsymbol{c}_{n}^{\prime}=$ $\left(c_{n, 0}^{\prime}, c_{n, 1}^{\prime}, \ldots, c_{n, L-1}^{\prime}\right)=\boldsymbol{c}_{n}+(q / 2) \boldsymbol{x}_{1} \in C$ such that $\xi^{c_{n, j}-c_{n, i}}+\xi^{c_{n, j}^{\prime}+c_{n, i}^{\prime}}=0$. Similarly if $i_{m} \neq j_{m}$, then for any sequence $\boldsymbol{c}_{n} \in C$, there exists $\boldsymbol{c}_{n}^{\prime}=\boldsymbol{c}_{n}+(q / 2) \boldsymbol{x}_{m} \in C$ such that $\xi^{c_{n, j}-c_{n, i}}+\xi^{c_{n, j}^{\prime}+c_{n, i}^{\prime}}=0$.

Lemma 9: [35, Lemma 5] Suppose $i_{\pi_{\alpha}(1)}=j_{\pi_{\alpha}(1)}$ for $\alpha=1,2, \ldots, k$. Let us consider three conditions:

(C1) $\hat{\alpha}$ is the largest integer satisfying $i_{\pi_{\alpha}(\beta)}=j_{\pi_{\alpha}(\beta)}$ for $\alpha=1, \ldots, \hat{\alpha}-1$ and $\beta=1, \ldots, m_{\alpha}$.

(C2) $\hat{\beta}$ is the smallest integer such that $i_{\pi_{\hat{\alpha}}(\hat{\beta})} \neq j_{\pi_{\hat{\alpha}}(\hat{\beta})}$.

(C3) Let $i^{\prime}$ and $j^{\prime}$ be integers which differ from $i$ and $j$, respectively, in only one position $\pi_{\hat{\alpha}}(\hat{\beta}-1)$. That is, $i_{\pi_{\hat{\alpha}}(\hat{\beta}-1)}^{\prime}=1-i_{\pi_{\hat{\alpha}}(\hat{\beta}-1)}$ and $j_{\pi_{\hat{\alpha}(\hat{\beta}-1)}^{\prime}}^{\prime}=1-j_{\pi_{\hat{\alpha}}(\hat{\beta}-1)}$.

If the above conditions are all satisfied, we have

$$
f_{j}-f_{i}-f_{j^{\prime}}+f_{i^{\prime}} \equiv q / 2 \quad(\bmod q) .
$$

Proof of Theorem 3: Here we are going to prove that $C$ is a GCS of size $2^{k+1}$ and of length $2^{m-1}+2^{t}$. To this end, it is sufficient to show

$$
\begin{aligned}
\rho(C ; u) & =\sum_{n=0}^{2^{k+1}-1} \rho\left(\boldsymbol{c}_{n} ; u\right) \\
& =\sum_{n=0}^{2^{k+1}-1} \sum_{i=0}^{L-1-u} \xi^{c_{n, i+u}-c_{n, i}} \\
& =\sum_{i=0}^{L-1-u} \sum_{n=0}^{2^{k+1}-1} \xi^{c_{n, i+u}-c_{n, i}}=0
\end{aligned}
$$

for $0<u<2^{m-1}+2^{t}-1$. For any integer $i<2^{m}+$ $2^{t}-1-u$ with binary representation $\left(i_{1}, i_{2}, \ldots, i_{m}\right)$, we let $j=i+u$ with binary representation $\left(j_{1}, j_{2}, \ldots, j_{m}\right)$. Then, we will show that (7) holds by considering the following four cases.

Case 1: If $i_{\pi_{\alpha}(1)} \neq j_{\pi_{\alpha}(1)}$ for some $\alpha \in\{1,2, \ldots, k\}$, according to Lemma 8 , we can obtain

$$
\sum_{n=0}^{2^{k+1}-1} \xi^{c_{n, j}-c_{n, i}}=0 .
$$

Case 2: In this case, we assume $i_{\pi_{\alpha}(1)}=j_{\pi_{\alpha}(1)}$ for all $\alpha \in\{1,2, \ldots, k\}$ and $i_{m}=j_{m}=0$. Here, there exist $\hat{\alpha}$ and $\hat{\beta}$ satisfying the conditions (C1) and (C2) in Lemma 9. Let $i^{\prime}$ and $j^{\prime}$ be integers fulfilling condition (C3) in Lemma 9. According to Lemma 9 , we have $j^{\prime}=i^{\prime}+u$ and $f_{j}-f_{i}-f_{j^{\prime}}+f_{i^{\prime}} \equiv q / 2$ $(\bmod q)$. Therefore,

$$
\begin{aligned}
c_{n, j}-c_{n, i}-c_{n, j^{\prime}}+c_{n, i^{\prime}} & =f_{j}-f_{i}-f_{j^{\prime}}+f_{i^{\prime}} \\
& \equiv q / 2(\bmod q) .
\end{aligned}
$$

Then the above equation implies

$$
\xi^{c_{n, j}-c_{n, i}}+\xi^{c_{n, j^{\prime}}-c_{n, i^{\prime}}}=0 .
$$

Case 3: In this case, suppose $i_{\pi_{\alpha}(1)}=j_{\pi_{\alpha}(1)}$ for all $\alpha \in\{1,2, \ldots, k\}$ and $i_{m}=j_{m}=1$ which means $2^{m-1} \leq$ $i, j \leq 2^{m-1}+2^{t}-1$. Here, there exist $\hat{\alpha}$ and $\hat{\beta}$ satisfying the conditions $(\mathrm{C} 1)$ and $(\mathrm{C} 2)$ in Lemma 9. Then, we will have $\pi_{\hat{\alpha}}(\hat{\beta}) \leq t$. Suppose not, we assume $\pi_{\hat{\alpha}}(\hat{\beta})>t$. Hence, we obtain that $i_{s}=j_{s}$ for $s=1,2, \ldots, t$ since $\left\{\pi_{1}(1), \ldots, \pi_{1}\left(m_{1}\right), \pi_{2}(1), \ldots, \pi_{\alpha^{\prime}}\left(\beta^{\prime}\right)\right\}=\{1,2, \ldots, t\}$. According to Lemma 6 , we have $j \geq i+2^{t} \geq 2^{m-1}+2^{t}$ which contradicts the assumption. Thus, we have $\pi_{\hat{\alpha}}(\hat{\beta}) \leq t$. Then let $i^{\prime}$ and $j^{\prime}$ be integers fulfilling condition (C3) in Lemma 9. Since $\pi_{\hat{\alpha}}(\hat{\beta}) \leq t$, we also have $\pi_{\hat{\alpha}}(\hat{\beta}-1) \leq t$. According to Lemma 7 , we obtain $2^{m-1} \leq i^{\prime}, j^{\prime} \leq 2^{m-1}+2^{\pi_{\hat{\alpha}}(\hat{\beta}-1)}-$ $1 \leq 2^{m-1}+2^{t}-1$. Therefore, for any integers $i$ and $j$ with $2^{m-1} \leq i, j \leq 2^{m-1}+2^{t}-1$, there exist integers $i^{\prime}, j^{\prime} \leq 2^{m-1}+2^{t}-1$ and $j^{\prime}=i^{\prime}+u$. Therefore, we obtain

$$
\xi^{c_{n, j}-c_{n, i}}+\xi^{c_{n, j^{\prime}}-c_{n, i^{\prime}}}=0 \text {. }
$$

according to Lemma 9.

Case 4: If $i_{m} \neq j_{m}$, according to Lemma 8, we also have

$$
\sum_{n=0}^{2^{k+1}-1} \xi^{c_{n, j}-c_{n, i}}=0
$$

According to Case 1 to Case 4 , we can confirm that $C$ is a GCS of length $L=2^{m-1}+2^{t}$.

\section{APPENDIX B \\ PROOF OF THEOREM 4}

Lemma 10: Let $\boldsymbol{x}_{n_{1}}, \boldsymbol{x}_{n_{2}}, \ldots, \boldsymbol{x}_{n_{k}}$ be the sequences corresponding to Boolean functions $x_{n_{1}}, x_{n_{2}}, \ldots, x_{n_{k}}$, respectively, where $n_{1}<n_{2}<\cdots<n_{k}$. Let a binary sequence $\boldsymbol{d}=\left(d_{0}, d_{1}, \ldots, d_{L-1}\right)=\boldsymbol{x}_{n_{1}} \oplus \boldsymbol{x}_{n_{2}} \oplus \cdots \oplus \boldsymbol{x}_{n_{k}}$ be the sum of $\boldsymbol{x}_{n_{1}}, \boldsymbol{x}_{n_{2}}, \ldots, \boldsymbol{x}_{n_{k}}$. If $2^{n_{1}} \mid L$, we can obtain that the Hamming weight of $\boldsymbol{d}$ is $L / 2$.

Proof: For any integers $i$, if $2^{n_{1}} \mid L$, then there exists an integer $i^{\prime}$ which is different from $i$ in only one position $n_{1}$, i.e., $\left(i_{1}^{\prime}, i_{2}^{\prime}, \ldots, i_{m}^{\prime}\right)=\left(i_{1}, i_{2}, \ldots, i_{n_{1}-1}, 1-i_{n_{1}}, i_{n_{1}+1}, \ldots, i_{m}\right)$. Thus,

$$
\begin{aligned}
d_{i^{\prime}}-d_{i} & =\left(i_{n_{1}}^{\prime} \oplus i_{n_{2}}^{\prime} \oplus \cdots \oplus i_{n_{k}}^{\prime}\right)-\left(i_{n_{1}} \oplus i_{n_{2}} \oplus \cdots \oplus i_{n_{k}}\right) \\
& \equiv i_{n_{1}}^{\prime}-i_{n_{1}} \equiv 1 \quad(\bmod 2)
\end{aligned}
$$

Therefore, $d_{i^{\prime}}=1-d_{i}$ which means there are half 0's and half 1 's for the binary sequence $\boldsymbol{d}$. That is, the Hamming weight of $\boldsymbol{d}$ is $L / 2$.

Proof of Theorem 4: We will show that any two distinct sets $C^{e}$ and $C^{p}$ where $0 \leq e \neq p \leq 2^{k^{\prime}}-1$ satisfy the ideal cross-correlation property. Let $\boldsymbol{c}_{n}^{e}=\left(c_{n, 0}^{e}, c_{n, 1}^{e}, \ldots, c_{n, L-1}^{e}\right) \in$ 
$C^{e}$ and $\boldsymbol{c}_{n}^{p}=\left(c_{n, 0}^{p}, c_{n, 1}^{p}, \ldots, c_{n, L-1}^{p}\right) \in C^{p}$ where $L=$ $2^{m-1}+2^{t}$. For $0 \leq u<L-1$, we have to show

$$
\begin{aligned}
\rho\left(C^{e}, C^{p} ; u\right) & =\sum_{n=0}^{2^{k+1}-1} \rho\left(\boldsymbol{c}_{n}^{e}, \boldsymbol{c}_{n}^{p} ; u\right) \\
& =\sum_{n=0}^{2^{k+1}-1} \sum_{i=0}^{L-1-u} \xi^{c_{n, i+u}^{e}-c_{n, i}^{p}} \\
& =\sum_{i=0}^{L-1-u} \sum_{n=0}^{2^{k+1}-1} \xi^{c_{n, i+u}^{e}-c_{n, i}^{p}}=0 .
\end{aligned}
$$

For any integer $i<2^{m-1}+2^{t}-1-u$ with binary representation $\left(i_{1}, i_{2}, \ldots, i_{m}\right)$, we let $j=i+u$ with binary representation $\left(j_{1}, j_{2}, \ldots, j_{m}\right)$. Then, we will show that (8) holds by considering the following four cases.

Case 1: Suppose $i_{\pi_{\alpha}(1)} \neq j_{\pi_{\alpha}(1)}$ for some $\alpha \in$ $\{1,2, \ldots, k\}$, according to Lemma 8 , we can obtain

$$
\sum_{n=0}^{2^{k+1}-1} \xi^{c_{n, j}^{e}-c_{n, i}^{p}}=0 .
$$

Case 2: In this case, we have $i_{\pi_{\alpha}(1)}=j_{\pi_{\alpha}(1)}$ for all $\alpha \in$ $\{1,2, \ldots, k\}$ and $i_{m}=j_{m}=0$. Let $\hat{\alpha}, \hat{\beta}, i^{\prime}$, and $j^{\prime}$ be given as those in Case 2 of the proof of Theorem 3. Similarly, according to Lemma 9 , we have $f_{j}-f_{i}-f_{j^{\prime}}+f_{i^{\prime}} \equiv q / 2(\bmod q)$. Thus,

$$
\xi^{c_{n, j}^{e}-c_{n, i}^{p}}+\xi^{c_{n, j^{\prime}}^{e}-c_{n, i^{\prime}}^{p}}=0 .
$$

Case 3: In this case, we have $i_{\pi_{\alpha}(1)}=j_{\pi_{\alpha}(1)}$ for all $\alpha \in$ $\{1,2, \ldots, k\}$ and $i_{m}=j_{m}=1$. Let $\hat{\alpha}, \hat{\beta}, i^{\prime}$, and $j^{\prime}$ be given as those in Case 3 of the proof of the Theorem 3. According to Lemma 9 , we also have $f_{j}-f_{i}-f_{j^{\prime}}+f_{i^{\prime}} \equiv q / 2(\bmod q)$. Then,

$$
\xi^{c_{n, j}^{e}-c_{n, i}^{p}}+\xi^{c_{n, j^{\prime}}^{e}-c_{n, i^{\prime}}^{p}}=0 .
$$

Case 4: Suppose $i_{m} \neq j_{m}$, according to Lemma 8, we have

$$
\sum_{n=0}^{2^{k+1}-1} \xi^{c_{n, j}^{e}-c_{n, i}^{p}}=0 .
$$

Combining these four cases, we can obtain that equation (8) holds for $0<u<L-1$. Now, it remains to show that for $u=0$,

$$
\begin{aligned}
\rho\left(C^{e}, C^{p} ; 0\right) & =\sum_{n=0}^{2^{k+1}-1} \rho\left(\boldsymbol{c}_{n}^{e}, \boldsymbol{c}_{n}^{p} ; 0\right) \\
& =\sum_{n=0}^{2^{k+1}-1} \sum_{i=0}^{L-1} \xi^{c_{n, i}^{e}-c_{n, i}^{p}}=0 .
\end{aligned}
$$

For any integer $n<2^{k+1}$, we have

$$
\boldsymbol{c}_{n}^{e}-\boldsymbol{c}_{n}^{p} \equiv \frac{q}{2} \boldsymbol{d} \quad(\bmod q)
$$

where

$$
\begin{aligned}
\boldsymbol{d}= & \left(e_{1} \oplus p_{1}\right) \boldsymbol{x}_{\pi_{1}\left(m_{1}\right)} \oplus\left(e_{2} \oplus p_{2}\right) \boldsymbol{x}_{\pi_{2}\left(m_{2}\right)} \oplus \cdots \\
& \oplus\left(e_{k^{\prime}} \oplus p_{k^{\prime}}\right) \boldsymbol{x}_{\pi_{k^{\prime}}\left(m_{k^{\prime}}\right)}
\end{aligned}
$$

where $\left(e_{1}, e_{2}, \ldots, e_{k^{\prime}}\right)$ and $\left(p_{1}, p_{2}, \ldots, p_{k^{\prime}}\right)$ are the binary representations of $e$ and $p$, respectively. We have $\boldsymbol{d} \neq \mathbf{0}$ since $e \neq p$. Let $s$ denote the minimum integer in the set $\left\{\pi_{1}\left(m_{1}\right), \pi_{2}\left(m_{2}\right), \ldots, \pi_{k^{\prime}}\left(m_{k^{\prime}}\right)\right\}$. Due to the condition (6), we have $\left\{\pi_{1}\left(m_{1}\right), \pi_{2}\left(m_{2}\right), \ldots, \pi_{k^{\prime}}\left(m_{k^{\prime}}\right)\right\} \subseteq\{1,2, \ldots, t\}$. Hence, we have $s \leq t$ implying $2^{s} \mid\left(2^{m-1}+2^{t}\right)$. According to Lemma 10, we can obtain that the Hamming weight of $\boldsymbol{d}$ is $L / 2$. Hence, for $i=0,1, \ldots, 2^{m-1}+2^{t}-1$, there are $L / 2$ pairs $\left(c_{n, i}^{e}, c_{n, i}^{p}\right)$ such that $\xi^{c_{n, i}^{e}-c_{n, i}^{p}}=\xi^{q / 2}=-1$ and $L / 2$ pairs $\left(c_{n, i}^{e}, c_{n, i}^{p}\right)$ such that $\xi^{c_{n, i}^{e}-c_{n, i}^{p}}=\xi^{0}=1$, so we have

$$
\rho\left(\boldsymbol{c}_{n}^{e}, \boldsymbol{c}_{n}^{p} ; 0\right)=\sum_{i=0}^{L-1} \xi^{c_{n, i}^{e}-c_{n, i}^{p}}=0
$$

which completes the proof.

\section{APPENDIX C \\ PROOF OF COROLlaRY 5}

Corollary 5 can be proved by following the similar logics in Theorem 4. Therefore, we only need to consider some other cases in this proof. We will show that any two distinct sets $C^{e}$ and $C^{p}$ where $0 \leq e \neq p \leq 2^{k}-1$ satisfy the ideal crosscorrelation property. For $0<u<L-1$ where $L=2^{m-1}+2^{t}$, we also have to show that the equation (8) is satisfied. We also consider four cases as follows.

Case 1: In this case, we have $i_{\pi_{\alpha}(1)} \neq j_{\pi_{\alpha}(1)}$ for some $\alpha \in\{1,2, \ldots, k\}$. Same as Case 1 of the proof of Theorem 4 , we can obtain

$$
\sum_{n=0}^{2^{k+1}-1} \xi^{c_{n, i+u}^{e}-c_{n, i}^{p}}=0 .
$$

Case 2: In this case, we have $i_{\pi_{\alpha}(1)}=j_{\pi_{\alpha}(1)}$ for all $\alpha \in$ $\{1,2, \ldots, k\}$ and $i_{m}=j_{m}=0$. Let $\hat{\alpha}, \hat{\beta}, i^{\prime}$, and $j^{\prime}$ be given as those in Case 2 of the proof of Theorem 3. Here, $y_{i}=$ $i_{m} i_{\pi_{k}\left(\beta^{\prime}\right)}+\left(1 \oplus i_{m}\right) i_{\pi_{k}\left(m_{k}\right)}=i_{\pi_{k}\left(m_{k}\right)}$. Also, $y_{i^{\prime}}=i_{\pi_{k}\left(m_{k}\right)}^{\prime}$, $y_{j}=j_{\pi_{k}\left(m_{k}\right)}$, and $y_{j^{\prime}}=j_{\pi_{k}\left(m_{k}\right)}^{\prime}$. According to Lemma 9, we have $f_{j}-f_{i}-f_{j^{\prime}}+f_{i^{\prime}} \equiv q / 2(\bmod q)$. Then,

$$
\begin{aligned}
c_{n, j}^{e}-c_{n, i}^{p}-c_{n, j^{\prime}}^{e}+c_{n, i^{\prime}}^{p} & \left(f_{j}+\frac{q}{2} \sum_{\alpha=1}^{k-1} e_{\alpha} j_{\pi_{\alpha}\left(m_{\alpha}\right)}+\frac{q}{2} e_{k} y_{j}\right) \\
& -\left(f_{i}+\frac{q}{2} \sum_{\alpha=1}^{k-1} p_{\alpha} i_{\pi_{\alpha}\left(m_{\alpha}\right)}+\frac{q}{2} p_{k} y_{i}\right) \\
& -\left(f_{j^{\prime}}+\frac{q}{2} \sum_{\alpha=1}^{k-1} e_{\alpha} j_{\pi_{\alpha}\left(m_{\alpha}\right)}^{\prime}+\frac{q}{2} e_{k} y_{j^{\prime}}\right) \\
+ & \left(f_{i^{\prime}}+\frac{q}{2} \sum_{\alpha=1}^{k-1} p_{\alpha} i_{\pi_{\alpha}\left(m_{\alpha}\right)}^{\prime}+\frac{q}{2} p_{k} y_{i^{\prime}}\right) \\
= & \left(f_{j}+\frac{q}{2} \sum_{\alpha=1}^{k-1} e_{\alpha} j_{\pi_{\alpha}\left(m_{\alpha}\right)}+\frac{q}{2} e_{k} j_{\pi_{k}\left(m_{k}\right)}\right) \\
& -\left(f_{i}+\frac{q}{2} \sum_{\alpha=1}^{k-1} p_{\alpha} i_{\pi_{\alpha}\left(m_{\alpha}\right)}+\frac{q}{2} p_{k} i_{\pi_{k}\left(m_{k}\right)}\right) \\
& -\left(f_{j^{\prime}}+\frac{q}{2} \sum_{\alpha=1}^{k-1} e_{\alpha} j_{\pi_{\alpha}\left(m_{\alpha}\right)}^{\prime}+\frac{q}{2} e_{k} j_{\pi_{k}\left(m_{k}\right)}^{\prime}\right)
\end{aligned}
$$




$$
\begin{aligned}
& +\left(f_{i^{\prime}}+\frac{q}{2} \sum_{\alpha=1}^{k-1} p_{\alpha} i_{\pi_{\alpha}\left(m_{\alpha}\right)}^{\prime}+\frac{q}{2} p_{k} i_{\pi_{k}\left(m_{k}\right)}^{\prime}\right) \\
& =f_{j}-f_{i}-f_{j^{\prime}}+f_{i^{\prime}} \\
& \equiv \frac{q}{2} \quad(\bmod q)
\end{aligned}
$$

since $i_{\pi_{\alpha}\left(m_{\alpha}\right)}=i_{\pi_{\alpha}\left(m_{\alpha}\right)}^{\prime}$ and $j_{\pi_{\alpha}\left(m_{\alpha}\right)}=j_{\pi_{\alpha}\left(m_{\alpha}\right)}^{\prime}$ for $\alpha=$ $1,2, \ldots, k$. This is because $i$ and $i^{\prime}$ differ in only one position $\pi_{\hat{\alpha}(\hat{\beta}-1)}$ and $\hat{\beta}-1<m_{\hat{\alpha}}$; so do $j$ and $j^{\prime}$. Hence, we can obtain

$$
\xi^{c_{n, i+u}^{e}-c_{n, i}^{p}}+\xi^{c_{n, i^{\prime}+u}^{e}-c_{n, i^{\prime}}^{p}}=0 .
$$

Case 3: In this case, we have $i_{\pi_{\alpha}(1)}=j_{\pi_{\alpha}(1)}$ for all $\alpha \in$ $\{1,2, \ldots, k\}$ and $i_{m}=j_{m}=1$. Different to Case 2, we have $y_{i}=i_{\pi_{k}\left(\beta^{\prime}\right)}, y_{i^{\prime}}=i_{\pi_{k}\left(\beta^{\prime}\right)}^{\prime}, y_{j}=j_{\pi_{k}\left(\beta^{\prime}\right)}$, and $y_{j^{\prime}}=j_{\pi_{k}\left(\beta^{\prime}\right)}^{\prime}$ instead. Let $\hat{\alpha}, \hat{\beta}, i^{\prime}$ and $j^{\prime}$ be given as those in Case 4 of the proof of Theorem 3. According to Lemma 9, we have $f_{j}-f_{i}-f_{j^{\prime}}+f_{i^{\prime}} \equiv q / 2(\bmod q)$. Thus,

$$
\begin{aligned}
& c_{n, j}^{e}-c_{n, i}^{p}-c_{n, j^{\prime}}^{e}+c_{n, i^{\prime}}^{p} \\
& =\left(f_{j}+\frac{q}{2} \sum_{\alpha=1}^{k-1} e_{\alpha} j_{\pi_{\alpha}\left(m_{\alpha}\right)}+\frac{q}{2} e_{k} j_{\pi_{k}\left(\beta^{\prime}\right)}\right) \\
& -\left(f_{i}+\frac{q}{2} \sum_{\alpha=1}^{k-1} p_{\alpha} i_{\pi_{\alpha}\left(m_{\alpha}\right)}+\frac{q}{2} p_{k} i_{\pi_{k}\left(\beta^{\prime}\right)}\right) \\
& -\left(f_{j^{\prime}}+\frac{q}{2} \sum_{\alpha=1}^{k-1} e_{\alpha} j_{\pi_{\alpha}\left(m_{\alpha}\right)}^{\prime}+\frac{q}{2} e_{k} j_{\pi_{k}\left(\beta^{\prime}\right)}^{\prime}\right) \\
& +\left(f_{i^{\prime}}+\frac{q}{2} \sum_{\alpha=1}^{k-1} p_{\alpha} i_{\pi_{\alpha}\left(m_{\alpha}\right)}^{\prime}+\frac{q}{2} p_{k} i_{\pi_{k}\left(\beta^{\prime}\right)}^{\prime}\right) \\
& =f_{j}-f_{i}-f_{j^{\prime}}+f_{i^{\prime}} \\
& +\frac{q}{2} e_{\alpha}^{\prime}\left(j_{\pi_{k}\left(\beta^{\prime}\right)}-j_{\pi_{k}\left(\beta^{\prime}\right)}^{\prime}\right)-\frac{q}{2} p_{\alpha}^{\prime}\left(i_{\pi_{k}\left(\beta^{\prime}\right)}-i_{\pi_{k}\left(\beta^{\prime}\right)}^{\prime}\right)
\end{aligned}
$$

where $i_{\pi_{\alpha}\left(m_{\alpha}\right)}=i_{\pi_{\alpha}\left(m_{\alpha}\right)}^{\prime}$ and $j_{\pi_{\alpha}\left(m_{\alpha}\right)}=j_{\pi_{\alpha}\left(m_{\alpha}\right)}^{\prime}$ for $\alpha=$ $1,2, \ldots, k$ as mentioned in Case 2. If $\pi_{k}\left(\beta^{\prime}\right)=\pi_{k}\left(m_{k}\right)$, then (9) can be further expressed as $c_{n, j}^{e}-c_{n, i}^{p}-c_{n, j^{\prime}}^{e}+c_{n, i^{\prime}}^{p}=$ $f_{j}-f_{i}-f_{j^{\prime}}+f_{i^{\prime}} \equiv q / 2(\bmod q)$. In another case, if $\pi_{k}\left(\beta^{\prime}\right) \neq$ $\pi_{k}\left(m_{k}\right)$ which implies $\beta^{\prime} \neq m_{k}$, then we shall have $\pi_{\hat{\alpha}}(\hat{\beta})<$ $\pi_{k}\left(\beta^{\prime}+1\right)$. Otherwise, $i_{s}=j_{s}$ for $s=1,2, \ldots, t$ since

$$
\begin{array}{r}
\left\{\pi_{1}(1), \pi_{1}(2), \ldots, \pi_{1}\left(m_{1}\right), \pi_{2}(1), \ldots, \pi_{2}\left(m_{2}\right),\right. \\
\left.\pi_{3}(1), \ldots, \pi_{k}(1), \ldots, \pi_{k}\left(\beta^{\prime}\right)\right\}=\{1,2, \ldots, t\}
\end{array}
$$

and $\pi_{k\left(\beta^{\prime}\right)}<\pi_{\hat{\alpha}}(\hat{\beta})$. According to Lemma 6 , we have $j \geq$ $i+2^{t} \geq 2^{m-1}+2^{t}$ which contradicts the assumption that $j<L=2^{m-1}+2^{t}$. Therefore, $\pi_{\hat{\alpha}}(\hat{\beta})<\pi_{k}\left(\beta^{\prime}+1\right)$ implying $\pi_{\hat{\alpha}}(\hat{\beta}-1)<\pi_{k}\left(\beta^{\prime}\right)$. Thus, we have $i_{\pi_{k}\left(\beta^{\prime}\right)}=i_{\pi_{k}\left(\beta^{\prime}\right)}^{\prime}$ and $j_{\pi_{k}\left(\beta^{\prime}\right)}=j_{\pi_{k}\left(\beta^{\prime}\right)}^{\prime}$. So we can obtain

$$
\begin{aligned}
c_{n, j}^{e}-c_{n, i}^{p}-c_{n, j^{\prime}}^{e}+c_{n, i^{\prime}}^{p} & =f_{j}-f_{i}-f_{j^{\prime}}+f_{i^{\prime}} \\
& \equiv \frac{q}{2}(\bmod q)
\end{aligned}
$$

implying

$$
\xi^{c_{n, i+u}^{e}-c_{n, i}^{p}}+\xi^{c_{n, i^{\prime}+u}^{e}-c_{n, i^{\prime}}^{p}}=0 .
$$

Case 4: In this case, we assume $i_{m} \neq j_{m}$. Same as Case 4 of the proof of Theorem 4, we have

$$
\sum_{n=0}^{2^{k+1}-1} \xi^{c_{n, i+u}^{e}-c_{n, i}^{p}}=0 .
$$

Combining these four cases, we can obtain that equation (8) holds for $0<u<L-1$. Similarly, it can also be obtained that equation (8) holds for $0<u<-L+1$. Now, it remains to show that

$$
\begin{aligned}
\rho\left(C^{e}, C^{p} ; 0\right) & =\sum_{n=0}^{2^{k+1}-1} \rho\left(\boldsymbol{c}_{n}^{e}, \boldsymbol{c}_{n}^{p} ; 0\right) \\
& =\sum_{n=0}^{2^{k+1}-1} \sum_{i=0}^{L-1} \xi^{c_{n, i}^{e}-c_{n, i}^{p}}=0 .
\end{aligned}
$$

For any integer $n<2^{k+1}$, we have

$$
\boldsymbol{c}_{n}^{e}-\boldsymbol{c}_{n}^{p} \equiv \frac{q}{2} \boldsymbol{d} \quad(\bmod q)
$$

where

$$
\begin{aligned}
\boldsymbol{d}= & \left(e_{1} \oplus p_{1}\right) \boldsymbol{x}_{\pi_{1}\left(m_{1}\right)} \oplus\left(e_{2} \oplus p_{2}\right) \boldsymbol{x}_{\pi_{2}\left(m_{2}\right)} \oplus \cdots \\
& \oplus\left(e_{k-1} \oplus p_{k-1}\right) \boldsymbol{x}_{\pi_{k-1}\left(m_{k-1}\right)} \\
& \oplus\left(e_{k} \oplus p_{k}\right)\left(\boldsymbol{x}_{m} \boldsymbol{x}_{\pi_{k}\left(\beta^{\prime}\right)}+\left(\mathbf{1} \oplus \boldsymbol{x}_{m}\right) \boldsymbol{x}_{\pi_{k}\left(m_{k}\right)}\right)
\end{aligned}
$$

where $\left(e_{1}, e_{2}, \ldots, e_{k}\right)$ and $\left(p_{1}, p_{2}, \ldots, p_{k}\right)$ are the binary representations of $e$ and $p$, respectively. We have $\boldsymbol{d} \neq \mathbf{0}$ since $e \neq p$. Here, we consider two cases to show that the Hamming weight of $\boldsymbol{d}$ is $L / 2$.

(i). For $e_{k} \oplus p_{k}=0$, we have

$$
\begin{aligned}
\boldsymbol{d}= & \left(e_{1} \oplus p_{1}\right) \boldsymbol{x}_{\pi_{1}\left(m_{1}\right)} \oplus\left(e_{2} \oplus p_{2}\right) \boldsymbol{x}_{\pi_{2}\left(m_{2}\right)} \oplus \cdots \\
& \oplus\left(e_{k-1} \oplus p_{k-1}\right) \boldsymbol{x}_{\pi_{k-1}\left(m_{k-1}\right)} .
\end{aligned}
$$

Then, arguing as in Case 4 of the proof of Theorem 4, we obtain the same result that $\boldsymbol{d}$ has Hamming weight $L / 2$.

(ii). For $e_{k} \oplus p_{k}=1$, we have

$$
\begin{aligned}
\boldsymbol{d}= & \left(e_{1} \oplus p_{1}\right) \boldsymbol{x}_{\pi_{1}\left(m_{1}\right)} \oplus\left(e_{2} \oplus p_{2}\right) \boldsymbol{x}_{\pi_{2}\left(m_{2}\right)} \oplus \cdots \\
& \oplus\left(e_{k-1} \oplus p_{k-1}\right) \boldsymbol{x}_{\pi_{k-1}\left(m_{k-1}\right)} \\
& \oplus \boldsymbol{x}_{m} \boldsymbol{x}_{\pi_{k}\left(\beta^{\prime}\right)} \oplus\left(\mathbf{1} \oplus \boldsymbol{x}_{m}\right) \boldsymbol{x}_{\pi_{k}\left(m_{k}\right)} .
\end{aligned}
$$

We express the sequence $\boldsymbol{d}$ into two parts, i.e., $\boldsymbol{d}=$ $\left(\boldsymbol{d}_{1}, \boldsymbol{d}_{2}\right)$ where $\boldsymbol{d}_{1}=\left(d_{0}, d_{1}, \ldots, d_{2^{m}-1}\right)$ and $\boldsymbol{d}_{2}=$ $\left(d_{2^{m}}, d_{2^{m}+1}, \ldots, d_{2^{m}+2^{t}-1}\right)$. For any positive integer $0 \leq i \leq$ $2^{m}-1$, we have $i_{m}=0$ implying

$$
\begin{aligned}
\boldsymbol{d}_{1}= & \left(e_{1} \oplus p_{1}\right) \boldsymbol{x}_{\pi_{1}\left(m_{1}\right)} \oplus\left(e_{2} \oplus p_{2}\right) \boldsymbol{x}_{\pi_{2}\left(m_{2}\right)} \oplus \cdots \\
& \oplus\left(e_{\alpha^{\prime}-1} \oplus p_{k-1}\right) \boldsymbol{x}_{\pi_{k-1}\left(m_{k-1}\right)} \oplus \boldsymbol{x}_{\pi_{k}\left(m_{k}\right)} .
\end{aligned}
$$

We have $\pi_{\alpha}\left(m_{\alpha}\right) \leq m-1$ for $\alpha=1,2, \ldots, k$, so $2^{\pi_{\alpha}\left(m_{\alpha}\right)} \mid 2^{m-1}$. According to Lemma 10, it can be obtained that the Hamming weight of $\boldsymbol{d}_{1}$ is half length, i.e., $2^{m-2}$.

For any positive integer $i$ with $2^{m} \leq i \leq 2^{m}+2^{t}-1$, we have $i_{m}=1$. Therefore, $\boldsymbol{d}_{2}$ can be expressed as

$$
\begin{aligned}
\boldsymbol{d}_{2}= & \left(e_{1} \oplus p_{1}\right) \boldsymbol{x}_{\pi_{1}\left(m_{1}\right)} \oplus\left(e_{2} \oplus p_{2}\right) \boldsymbol{x}_{\pi_{2}\left(m_{2}\right)} \oplus \cdots \\
& \oplus\left(e_{k-1} \oplus p_{k-1}\right) \boldsymbol{x}_{\pi_{k-1}\left(m_{k-1}\right)} \oplus \boldsymbol{x}_{\pi_{k}\left(\beta^{\prime}\right)} .
\end{aligned}
$$

Since $\quad\left\{\pi_{1}\left(m_{1}\right), \pi_{2}\left(m_{2}\right), \cdots, \pi_{k-1}\left(m_{k-1}\right), \pi_{k}\left(\beta^{\prime}\right)\right\} \quad \in$ $\{1,2, \ldots, t\}$, we have $\pi_{k}\left(\beta^{\prime}\right) \leq t$ and $\pi_{\alpha}\left(m_{\alpha}\right) \leq t$ for 
$\alpha=1,2, \ldots, k-1$. Thus, it can be obtained that the Hamming weight of $\boldsymbol{d}_{2}$ is $2^{t} / 2=2^{t-1}$ according to Lemma 10 .

Hence, the Hamming weight of $\boldsymbol{d}$ is $2^{m-2}+2^{t-1}=L / 2$. There are $L / 2$ pairs $\left(c_{n, i}^{e}, c_{n, i}^{p}\right)$ such that $\xi^{c_{n, i}^{e}-c_{n, i}^{p}}=\xi^{q / 2}=$ -1 and $L / 2$ pairs $\left(c_{n, i}^{e}, c_{n, i}^{p}\right)$ such that $\xi^{c_{n, i}^{e}-c_{n, i}^{p}}=\xi^{0}=1$, so we have

$$
\rho\left(\boldsymbol{c}_{n}^{e}, \boldsymbol{c}_{n}^{p} ; 0\right)=\sum_{i=0}^{2^{m}-1} \xi^{c_{n, i}^{e}-c_{n, i}^{p}}=0
$$

which completes the proof.

\section{ACKNOWLEDGMENT}

The authors would like to thank the anonymous reviewers and Associate Editor Ryan Gabrys for their valuable comments and suggestions.

\section{REFERENCES}

[1] M. J. E. Golay, "Complementary series," IRE Trans. Inf. Theory, vol. IT-7, pp. 82-87, Apr. 1961.

[2] C.-C. Tseng and C. L. Liu, "Complementary sets of sequences," IEEE Trans. Inf. Theory, vol. IT-18, no. 5, pp. 644-652, Sep. 1972.

[3] N. Suehiro and M. Hatori, " $N$-shift cross-orthogonal sequences," IEEE Trans. Inf. Theory, vol. 34, no. 1, pp. 143-146, Jan. 1988.

[4] S.-M. Tseng and M. R. Bell, "Asynchronous multicarrier DS-CDMA using mutually orthogonal complementary sets of sequences," IEEE Trans. Commun., vol. 48, pp. 53-59, Jan. 2000.

[5] H.-H. Chen, J.-F. Yeh, and N. Suehiro, "A multicarrier CDMA architecture based on orthogonal complete complementary codes for new generations of wideband wireless communications," IEEE Commun. Mag., vol. 39, pp. 126-134, Oct. 2001.

[6] H.-H. Chen, The Next Generation CDMA Technologies. Wiley, 2007.

[7] Z. Liu, Y. L. Guan, and U. Parampalli, "New complete complementary codes for peak-to-mean power control in multi-carrier CDMA," IEEE Trans. Commun., vol. 62, no. 3, pp. 1105-1113, Mar. 2014

[8] Z. Liu, Y. L. Guan, and H.-H. Chen, "Fractional-delay-resilient receiver design for interference-free MC-CDMA communications based on complete complementary codes," IEEE Trans. Wireless Commun., vol. 14, no. 3, pp. 1226-1236, Mar. 2015.

[9] S. Wang and A. Abdi, "MIMO ISI channel estimation using uncorrelated Golay complementary sets," IEEE Trans. Veh. Technol., vol. 56, no. 5, pp. 3024-3039, Sep. 2007.

[10] S. F. Li, J. Chen, and L. Q. Zhang, "Optimisation of complete complementary codes in MIMO radar system," Electron. Lett., vol. 46, no. 16 , pp. 1157-1159, Aug. 2010

[11] J. Tang, N. Zhang, Z. Ma, and B. Tang, "Construction of Doppler resilient complete complementary code in MIMO radar," IEEE Trans. Signal Process., vol. 62, no. 18, pp. 4704-4712, Sep. 2014.

[12] C.-Y. Chen, Y.-J. Min, K.-Y. Lu, and C.-c. Chao, "Cell search for cellbased OFDM systems using quasi complete complementary codes," in Proc. IEEE Int. Conf. Commun., Beijing, China, May 2008, pp. 48404844.

[13] T. Kojima, T. Tachikawa, A. Oizumi, Y. Yamaguchi, and U. Parampalli, "A disaster prevention broadcasting based on data hiding scheme using complete complementary codes," in Proc. Int. Symp. on Inform. Theory and its Applicat., Melbourne, Australia, Oct. 2014, pp. 45-49.

[14] J. A. Davis and J. Jedwab, "Peak-to-mean power control in OFDM, Golay complementary sequences, and Reed-Muller codes," IEEE Trans. Inf. Theory, vol. 45, no. 7, pp. 2397-2417, Nov. 1999.

[15] K. G. Paterson, "Generalized Reed-Muller codes and power control in OFDM modulation," IEEE Trans. Inf. Theory, vol. 46, no. 1, pp. 104120, Jan. 2000.

[16] Y. Li and W. B. Chu, "More Golay sequences," IEEE Trans. Inf. Theory, vol. 51, no. 3, pp. 1141-1145, Mar. 2005.

[17] Z. Liu, U. Parampalli, and Y. L. Guan, "On even-period binary Zcomplementary pairs with large ZCZs," IEEE Signal Process. Lett., vol. 21, no. 3, pp. 284-287, Mar. 2014.

[18] — " "Optimal odd-length binary Z-complementary pairs," IEEE Trans. Inf. Theory, vol. 60, no. 9, pp. 5768-5781, Sep. 2014.
[19] K.-U. Schmidt, "Complementary sets, generalized Reed-Muller codes, and power control for OFDM," IEEE Trans. Inf. Theory, vol. 53, no. 2, pp. 808-814, Feb. 2007.

[20] M. G. Parker and C. Tellambura, "A construction for binary sequence sets with low peak-to-average power ratio," in Proc. IEEE Int. Symp. Inform. Theory, Lausanne, Switzerland, Jun. 2003, p. 239.

[21] W. Chen and C. Tellambura, "Identifying a class of multiple shift complementary sequences in the second order cosets of the first order Reed-Muller codes," in Proc. IEEE Int. Conf. Commun., Seoul, Korea, May 2005, pp. 618-621.

[22] C.-Y. Chen, C.-H. Wang, and C.-c. Chao, "Complementary sets and Reed-Muller codes for peak-to-average power ratio reduction in OFDM,' in Proc. 16th Int. Symp. AAECC, LNCS 3857, Las Vegas, NV, Feb. 2006, pp. 317-327.

[23] A. Rathinakumar and A. K. Chaturvedi, "Complete mutually orthogonal Golay complementary sets from Reed-Muller codes," IEEE Trans. Inf. Theory, vol. 54, pp. 1339-1346, Mar. 2008.

[24] C.-Y. Chen, C.-H. Wang, and C.-c. Chao, "Complete complementary codes and generalized Reed-Muller codes," IEEE Commun. Lett., vol. 12 , pp. 849-851, Nov. 2008.

[25] C.-Y. Chen, "Complementary sets of non-power-of-two length for peakto-average power ratio reduction in OFDM," IEEE Trans. Inf. Theory, vol. 62, no. 12, pp. 7538-7545, Dec. 2016.

[26] _ - "A new construction of Golay complementary sets of non-powerof-two length based on Boolean functions," in Proc. IEEE Wireless Commun. and Netw. Conf., San Francisco, CA, Mar. 2017, pp. 1-6.

[27] _ "A novel construction of complementary sets with flexible lengths based on Boolean functions," IEEE Commun. Lett., vol. 22, no. 2, pp. 260-263, Feb. 2018.

[28] S. Das, S. Budišin, S. Majhi, Z. Liu, and Y. L. Guan, "A multiplier-free generator for polyphase complete complementary codes," IEEE Trans. Signal Process., vol. 66, no. 5, pp. 1184-1196, Mar. 2018.

[29] S. Das, S. Majhi, and Z. Liu, "A novel class of complete complementary codes and their applications for APU matrices," IEEE Signal Process. Lett., vol. 25, no. 9, pp. 1300-1304, Sep. 2018.

[30] S. Das, S. Majhi, S. Budišin, and Z. Liu, "A new construction framework for polyphase complete complementary codes with various lengths," IEEE Trans. Signal Process., vol. 67, no. 10, pp. 2639-2648, May 2019.

[31] Z. Liu and Y. L. Guan, "16-QAM almost-complementary sequences with low PMEPR," IEEE Trans. Commun., vol. 64, no. 2, pp. 668-679, Feb. 2016.

[32] C. Han, N. Suehiro, and T. Hashimoto, "A systematic framework for the construction of optimal complete complementary codes," IEEE Trans. Inf. Theory, vol. 57, no. 9, pp. 6033-6042, Sep. 2011.

[33] K. G. Paterson, "Generalized Reed-Muller codes and power control in OFDM modulation," IEEE Trans. Inf. Theory, vol. 46, pp. 104-120, Jan. 2000.

[34] F. J. MacWilliams and N. J. A. Sloane, The Theory of Error Correcting Codes. Amsterdam, The Netherlands: North-Holland, 1977.

[35] S.-W. Wu and C.-Y. Chen, "Optimal Z-complementary sequence sets with good peak-to-average power-ratio property," IEEE Signal Process. Lett., vol. 25, no. 10, pp. 1500-1504, Oct. 2018.

Shing-Wei Wu received his B.S. degree in electrical engineering from the Chinese Culture University, Taipei, in 2016 and M.S. degree in engineering science from the National Cheng Kung University, Tainan, Taiwan in 2018. Currently, he is pursuing the Ph.D. degree in engineering science from the National Cheng Kung University, Tainan, Taiwan. His research interests include sequence design and its applications on communications.

Chao-Yu Chen (M'17) received the B.S. degree in electrical engineering from the National Tsing Hua University (NTHU), Hsinchu, in 2000 and the M.S and $\mathrm{Ph} . \mathrm{D}$. degrees in communications engineering from NTHU, Hsinchu, in 2002 and 2009, respectively, under the supervision of Prof. Chi-chao Chao. 
He was a visiting Ph.D. student with the University of California, Davis, from 2008 to 2009 (with Prof. Shu Lin). From 2009 to 2016, he was a technical manager in Communication System Design division, Mediatek Inc., Hsinchu, Taiwan. From July 2018 to August 2018, he was with the University of California, Davis, as a visiting scholar (with Prof. Shu Lin). Since February 2016, he has been a Faculty Member with the National Cheng Kung University, Tainan, Taiwan, where he is currently an assistant professor at the Department of Engineering Science. His current research interests include sequence design, error-correcting codes, digital communications, and wireless networks.

Dr. Chen was a recipient of the 15th Y. Z. Hsu Science Paper Award administered by Far Eastern Y. Z. Hsu Science and Technology Memorial Foundation, Taiwan, in 2017 and the Best Paper Award for Young Scholars by the IEEE Information Theory Society Taipei Chapter and the IEEE Communications Society Taipei/Tainan Chapter in 2018. Since January 2019, he serves as the Vice Chair of the IEEE Information Theory Society Tainan Chapter.

Zilong Liu received his PhD (2014) from School of Electrical and Electronic Engineering, Nanyang Technological University (NTU), Master Degree (2007) in the Department of Electronic Engineering from Tsinghua University, and Bachelor Degree (2004) in the School of Electronics and Information Engineering from Huazhong University of Science and Technology (HUST). He is a Lecturer at the School of Computer Science and Electronic Engineering, University of Essex. From Jan. 2018 to Nov. 2019, he was a Senior Research Fellow at the Institute for Communication Systems (ICS), Home of the 5G Innovation Centre (5GIC), University of Surrey. Prior to his career in UK, he spent 9.5 years in NTU, Singapore, first as a Research Associate (Jul. 2008 to Oct. 2014) and then a Research Fellow (Nov. 2014 to Dec. 2018). His research lies in the interplay of communication, coding, signal processing, and a wide range of mathematical tools (e.g., number theory, abstract algebra, and convex optimization). He is extremely in attacking research problems that arise in practical communication systems. Details of his research can be found at: https://sites.google.com/site/zilongliu2357. 